\title{
AS QUESTÕES MACROSSOCIAIS DAS DROGAS E O SABERES DOS ESTUDANTES DE ENFERMAGEM
}

\author{
The Macrosocial Issues of the Drugs and the \\ Knowledge of the Nursing Students \\ Las Cuestiones Macrosociales de las Drogas y \\ los Saberes de los Estudiantes de Enfermería
}

Bárbara Rodrigues Carvalho Cordeiro ${ }^{1}$

Bruna Kelly de Jesus Lemos ${ }^{4}$

\author{
Helen Balthazar de Lima² \\ Tiago Ribeiro Lemos $^{5}$
}

Luana dos Santos Vasconcellos Lima ${ }^{3}$ Gertrudes Teixeira Lopes ${ }^{6}$

\section{Resumo}

0 objeto são as associações feitas entre o fenômeno das drogas e as questões macrossociais e profissionais na formação do estudante de graduação. Objetivos: descrever as associações feitas entre o fenômeno das drogas e as políticas de saúde internacionais, nacionais e locais e analisar a percepção dos estudantes sobre a atuação e o interesse em relação às drogas. Pesquisa quantitativa, apresenta resultados parciais de 16 faculdades de Enfermagem do Rio de Janeiro; a amostra é de 181 graduandos. Utilizou-se um questionário com escalas sobre este conhecimento, agregando três opções (concordo, indiferente e discordo). Os dados foram analisados com auxílio dos programas Epi-Info e Excell e apresentados em tabelas. Os resultados demonstraram que as políticas e programas nacionais e locais sobre o fenômeno das drogas foram abordados na graduação segundo a maioria dos estudantes, bem como tratar-se de um tema de interesse para a atuação do enfermeiro. Entretanto, em relação à abordagem das questões internacionais, houve uma distribuição semelhante entre as alternativas propostas.

Palavras-chave: Enfermagem. Estudantes de Enfermagem. Drogas ilícitas. Bebidas Alcoólicas. Conhecimento.

\section{Abstract}

The associations which were made between Drugs phenomenon and macro socials and professionals issues around graduating students formation are the subject. Goals: describing the associations that were made between drugs phenomenon and also international, national and local politics and Health; and analyzing students' perception about acting and interest by Drugs. A quantitative research presents partial results of sixteen Nursing Colleges from Rio de Janeiro; the sample is from 181 graduating students. Has been used a scale type questionnaire about this knowledge putting together three options (I agree, I'm not interested, I don't agree). Those data were presented in table and analyzed with the programs: Epi-Info and Excel. The results demonstrated that, according to the majority of the students, the national and local politics and programs about Drugs phenomenon were faced during graduating and it is also an interesting issue at the nurses' acts. However, there was a similar distribution around the alternatives which were proposed during the approach to international issues.

\section{Resumen}

El objeto es las asociaciones hechas entre el fenómeno de las drogas y las cuestiones macrosociales y profesionales en la formación del estudiante de pret-grado. Objetivos: describir las asociaciones hechas entre el fenómeno de las drogas y las políticas de salud internacionales, nacionales y locales, y analizar la percepción de los estudiantes sobre la actuación y el interés en relación con las drogas. Investigación cuantitativa la cual presenta datos parciales de 16 facultades de enfermería de Rio de Janeiro; la muestra es de 181 alumnos de pretgrado. Se utilizó un cuestionariocon escala sobre estos conocimientos, conteniendo tres alternativas (estoy de acuerdo, indiferente y no estoy de acuerdo). Los datos fueron analizados con la ayuda del Programa Epi-Info y Excell y presentados en tablas. Los resultados muestraron quelas políticasy los programas nacionales ylocales sobre el fenómeno de las drogas fueron abordados en el curso de pret-grado según la mayoría de los estudiantes, además de se considerar uno asunto del interés para la actuación del enfermero. Sin embargo, con relación al abordaje de las cuestiones internacionales, hubo una distribución semejante entre las alternativas que fueron propuestas.
Keywords: Nursing. Students,Nursing. Street Drugs. Alcoholic Beverages. Knowledge.
Palabras clave:Enfermaría. Estudiantes de Enfermería. Drogas llícitas. Bebidas Alcohólicas. Conocimiento.

${ }^{1}$ Aluna do $6^{\circ}$ período de graduação da Faculdade de Enfermagem da UERJ. Bolsista de Iniciação Científica da FAPERJ.2.Aluna do $6^{\circ}$ período de graduação da Faculdade de Enfermagem da UERJ. Bolsista de Iniciação Científica da UERJ. ${ }^{3}$ Aluna do $6^{\circ}$ período de graduação da Faculdade de Enfermagem da UERJ. Voluntária do Projeto. ${ }^{4}$ Aluna do $6^{\circ}$ período de graduação da Faculdade de Enfermagem da UERJ. Bolsista de Iniciação Científica da FAPERJ. ${ }^{5}$ Aluno do $9^{\circ}$ período de graduação da Faculdade de Medicina da UFRJ. Bolsista de Apoio Técnico do CNPq. ${ }^{6}$ Professora Titular da Faculdade de Enfermagem da UERJ. Livre Docente e Doutora em Enfermagem. Pós-Doutorada na área de Álcool e Drogas. Procientista da UERJ. Pesquisadora do CNPq. Membro do Núcleo de Pesquisa em História da Enfermagem Brasileira (NUPHEBRAS) da EEAN/UFRJ e do Grupo de Estudos e Pesquisas em álcool e outras Drogas (GEPAD) da FENF/UERJ. 


\section{INTRODUÇÃO}

As universidades não podem ignorar as questões das sociedades nas quais estão inseridas e que as mantêm e tampouco aquelas que afetam a humanidade. Desta forma, deve estar claro que a liberdade acadêmica e a autonomia da universidade são indissociáveis de sua responsabilidade social. A riqueza e o desenvolvimento social das nações dependem da capacidade de gerar conhecimento, e as universidades representam um dos principais agentes institucionais geradores e aplicadores do conhecimento, junto com as empresas e os órgãos públicos de pesquisa'.

0 conhecimento pode ser definido como "certeza de que os fenômenos são reais e possuem características específicas". 0 termo real neste contexto é considerado "uma qualidade pertencente a fenômenos que reconhecemos terem um ser independente de nossa própria volição". 0 conhecimento é visto como componente de uma realidade que não é única para as várias sociedades. Dessa forma, ele é caracterizado pela sua relatividade social, referindo-se a contextos sociais específicos ${ }^{2: 247}$.

0 conhecimento é parte componente tanto das crenças quanto das atitudes desenvolvidas pelo indivíduo ${ }^{3}$. Assim considerando, o conhecimento gerado, seja na forma de ensino ou pesquisa, deve se configurar numa visão macro. Dentre os conhecimentos destacados na contemporaneidade incluem-se as questões referentes ao fenômeno das drogas. Neste aspecto, o ensino formal na área de Enfermagem sobre o uso e abuso de drogas parece não corresponder às reais necessidades que a temática vem impondo à sociedade nos últimos anos.

Sabe-se que os currículos de Enfermagem contemplam de alguma forma a abordagem do uso e abuso de substâncias lícitas e ilícitas; no entanto, este conteúdo é majoritariamente ministrado nas disciplinas que envolvem saúde mental, com uma carga horária que não permite habilitar o enfermeiro para o desempenho adequado de suas funções no que tange às medidas tanto de promoção como de prevenção, tratamento e inserção social dos usuários ${ }^{4}$.

Em relação aos usuários de álcool, as intervenções realizadas nos cuidados de enfermagem ainda não atingiram a resolutividade esperada por falta de embasamento teóricocientífico sobre 0 assunto ${ }^{4}$.

Nesse sentido, os enfermeiros, por lidarem diretamente com essa clientela, percebem mais as intervenções empregadas nos cuidados de enfermagem do que os docentes e alunos investigados ${ }^{5}$.

Diante da magnitude que o problema drogas suscita, tornase imperativo que os conteúdos a serem ministrados nos cursos de graduação em Enfermagem possam corresponder às exigências e às necessidades da população brasileira ${ }^{4}$.

Entende-se que um fenômeno com a amplitude das drogas não pode ser compreendido apenas por uma visão estreita e focalizada, precisa ser discutido em vários aspectos desde os mais globais, aos mais pontuais, para que se perceba realmente a dimensão do problema.

Nesta lógica, delineou-se como objeto deste estudo as associações feitas entre o fenômeno das drogas e as questões macrossociais e profissionais na formação do estudante de graduação. Para elucidar o objeto, elaboraram-se como questões norteadoras: Na graduação foram feitas associações entre o fenômeno das drogas e a política e saúde internacionais? Foram discutidos conteúdos sobre o fenômeno das drogas e as políticas nacionais antidrogas? Durante a formação acadêmica foram feitas relações entre a atuação do enfermeiro e o fenômeno das drogas? A formação atual dos estudantes de Enfermagem oferece conhecimento apropriado e desperta o interesse para que eles se tornem enfermeiros com desempenho profissional satisfatório diante do fenômeno das drogas?

Para responder a estas questões foram elaborados os seguintes objetivos: descrever as associações feitas entre 0 fenômeno das drogas e as políticas de saúde internacionais, nacionais e locais; analisar a percepção dos estudantes sobre a atuação e 0 interesse em relação às drogas

Este estudo pretende contribuir para reflexão dos órgãos formadores, no sentido de incorporarem conteúdos macro, micro e pontual nos currículos de Enfermagem, sobre o fenômeno das drogas, objetivando fortalecer o conhecimento e a discussão em torno desta temática. Pretende ainda fortalecer o conhecimento dos enfermeiros em que pese à dimensão que o fenômeno das drogas implica.

Este artigo integra ao Projeto de Pesquisa intitulado "Conhecimentos, Atitudes e Crenças dos Enfermeiros sobre o Fenômeno das Drogas Adquiridos na Graduação: Estudo desenvolvido no Estado do Rio de Janeiro" sob a coordenação da Prof ${ }^{a}$ Dra. Gertrudes Teixeira Lopes, do Grupo de Estudos e Pesquisas em Álcool e outras Drogas (GEPAD), e conta com apoio do CNPq , UERJ e FAPERJ.

\section{METODOLOGIA}

Trata-se de uma pesquisa integrada, interdisciplinar, que se utiliza da abordagem quantitativa do tipo descritiva. 0 estudo foi desenvolvido em 16 instituições privadas de ensino superior de Enfermagem, do Estado do Rio de Janeiro, que agregam 23 unidades de ensino, considerando que a Universidade Estácio de Sá incorpora oito unidades acadêmicas.

Para participar da amostra os estudantes deveriam atender aos seguintes critérios: ser aluno regularmente matriculado na instituição de ensino; ter cursado a Escola/Faculdade desde o início da graduação; ser aluno do último período acadêmico; desejar participar voluntariamente da pesquisa. Para obtenção dos dados dos estudantes nas diferentes instituições de ensino, entrou-se em contato, inicialmente por telefone ou e-mail, com os dirigentes das escolas para agendamento de encontros para apresentação do Projeto de Pesquisa.

Após a apresentação do projeto às instituições investigadas e aprovação do mesmo nas suas diferentes instâncias, foi feito contato com os professores indicados para o acompanhamento da execução da coleta dos dados, de acordo com suas diretrizes.

Junto a esses professores, foram agendados datas e locais para a aplicação do questionário, ocasiões em que foi apresentado aos alunos o projeto, dando-se as informações necessárias, solicitando-se a colaboração daqueles que concordaram em participar do estudo. 
0 dimensionamento da amostra para cada Instituição de Ensino consubstanciou na proporção de atributos, erro de estimativa pré-fixado em $10 \%$, nível de significância de $5 \%$ e amostragem aleatória sem reposição. A população é composta por 1.301 estudantes, e a amostra foi dimensionada em 352 alunos, correspondente às 23 unidades acadêmicas. Como se trata de resultados parciais, a amostra aqui analisada é de 181 acadêmicos que aceitaram participar desta fase da pesquisa mediante assinatura do Termo de Consentimento Livre e Esclarecido. 0 projeto foi submetido e aprovado (1309-CEP/HUPE) pelo Comitê de Ética do Hospital Universitário Pedro Ernesto - HUPE/UERJ.

Para a coleta de dados foi utilizado um questionário composto por três partes. Este artigo discute a segunda parte da escala que aborda o conhecimento dos estudantes sobre o fenômeno das drogas. As demais partes foram analisadas e desenvolvidas em outros artigos científicos. A escala foi submetida ao teste piloto com a avaliação de 76 estudantes do último período acadêmico de uma escola privada do Rio de Janeiro. As questões referentes ao conhecimento incorporam as variáveis: visão macro, que se direciona para a política e saúde internacionais, aspectos econômicos, sócio-culturais e a globalização; visão micro, que aborda os aspectos das políticas nacionais, a saúde em geral e atuação e o interesse do enfermeiro pela temática; visão pontual, que incorpora aspectos das políticas regionais e locais, totalizando 10 afirmativas, com três opções para respostas (concordo, indiferente e discordo).

A coleta dos dados ocorreu no período de abril a dezembro de 2006. Os dados foram analisados com apoio do programa estatístico Epi-Info, apresentado sob a forma de tabelas elaboradas com auxílio do programa Excel, e discutidos segundo a literatura correspondente.

\section{RESULTADOS E DISCUSSÃO}

Os dados obtidos neste recorte da pesquisa indicam que os aspectos internacional (visão macro), nacional (visão micro) e local (visão pontual) do tema drogas foram abordados com os graduandos de Enfermagem das escolas privadas pesquisadas, no Estado do Rio de Janeiro, o que se considera um avanço para a compreensão e a dimensionamento do fenômeno das drogas no conhecimento dos futuros profissionais de enfermagem.

A visão macro do fenômeno das drogas considera as dimensões das políticas internacionais, da saúde internacional, da globalização e questões sócio-econômico-cultural, conforme apresentado na Tabela 1.

\begin{tabular}{|c|c|c|c|c|c|c|}
\hline \multirow{3}{*}{ 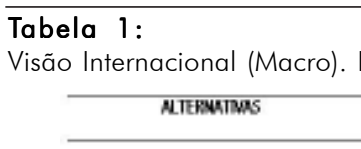 } & \multirow{3}{*}{\multicolumn{2}{|c|}{ 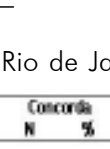 }} & \multicolumn{2}{|c|}{ eiro, 2007} & \multicolumn{2}{|c|}{7} \\
\hline & & & & mente & & \\
\hline & & & K & $\%$ & N & $\%$ \\
\hline 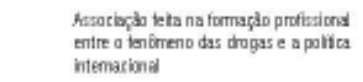 & $u$ & 40,9 & 64 & 35,4 & 43 & 23,7 \\
\hline 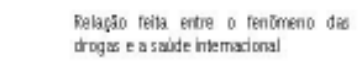 & 76 & 42,0 & 61 & 33,7 & 44 & 24,3 \\
\hline 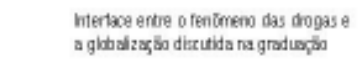 & 94 & תג, & 46 & 23,0 & 41 & 250 \\
\hline 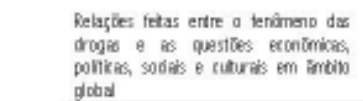 & 117 & 65 & 30 & 16 & 34 & 19 \\
\hline
\end{tabular}

Sob o prisma da globalização, a visão macro toma configurações de disseminadora das informações, produzindo o encurtamento do mundo global. Com estas características, pode assumir a condição de responsável pela rapidez na divulgação do conhecimento e das práticas condicionantes.

A análise da associação realizada entre a variável políica internacional e o fenômeno das drogas, durante a formação dos estudantes de Enfermagem, demonstra que 74 (40,9\%) concordam com esta alternativa; $64(35,4 \%)$ posicionaramse de modo indiferente e $43(23,7 \%)$ discordam desta afirmativa. A análise conjunta dos itens indiferente e discordante demonstra que 107 (59,2\%) acadêmicos que responderam ao instrumento de coleta de dados, mesmo estando no último período da graduação, ainda têm dúvidas ou não se posicionaram em relação ao assunto em pauta.

Os resultados evidenciam que apesar de a maioria optar pela variável concordo, houve uma certa aproximação com a variável indiferente e desta com discordo.

Conhecer e compreender como as políticas internacionais se configuram em torno do fenômeno das drogas leva 0 profissional a ter uma visão mais ampliada acerca do tema, contribuindo para que sua atuação seja conectada com o mundo.

Atualmente não se pode agir sem a consciência de que se está num mundo globalizado e que as ações se inter-relacionam e se complementam. Entretanto, também é importante compreender que estas políticas "regulam" a autonomia de seus signatários na medida em que estabelecem normas comuns e, não raro, uma abordagem comum a um fenômeno ainda por ser decifrado. É necessário ter consciência que no "mundo das drogas" as fronteiras não existem e as culturas se misturam 6 .

Estar em consonância com as iniciativas globais em torno do fenômeno das drogas parece um pré-requisito para que o profissional atue de tal modo que responda às necessidades da população a que assiste ${ }^{6}$.

Considerando a associação entre o fenômeno das drogas e a saúde internacional, os dados evidenciam que $76(42,0 \%)$ dos estudantes concordam com esta afirmativa; 61 (33,7\%) foram indiferentes e $44(24,3 \%)$ discordam. Estes resultados reafirmam certa fragilidade nos conteúdos apresentados durante a formação profissional dos enfermeiros, na medida em que a maioria se distribui entre as variáveis indiferente e discordo.

Nesta lógica, os resultados são incoerentes com o conceito de saúde internacional, que consiste em um conjunto de interações sanitárias que ocorrem em escala global, com as dimensões de toda a humanidade ${ }^{7}$.

Corroborando a afirmativa, outros autores afirmam que Saúde Internacional é:

Um processo dinâmico de interação, integração, equilíbrio de poder, interesses e tomada de decisões da comunidade internacional e nacional, quanto a questões e problemas relacionados com a saúde, que ocorram nos âmbitos nacional e internacional, e que tenham influência na esfera global e em sua relação com 0 processo de desenvolvimento de um país 8 .

É importante ressaltar que a saúde internacional tem adquirido importância significativa em função da crescente 
complexidade das relações internacionais com seus impactos nos sistemas de saúde. Essa interação fundamenta a necessidade de desenvolvimento e consolidação de uma tradição acadêmica e intelectual que sustente a geração de conhecimento, dando base para suas aplicações práticas ${ }^{9}$.

Analisando-se os dados referentes à interface entre 0 fenômeno das drogas e a globalização, pode-se observar que mais da metade dos acadêmicos, isto é, 94 (52,0\%), concordaram que durante a graduação foi apresentada a interface entre os fenômenos das drogas e a globalização, 46 (25,0\%) mantiveram-se indiferentes e 41 (23,0\%) discordaram.

0 cenário mundial vem apresentando mudanças a cada dia, gerando incertezas sobre o processo de integração econômica e social. Em conseqüência do processo de globalização, estas incertezas influenciam e reconfiguram os papéis e as relações entre as nações. Neste contexto, emerge de forma exacerbada o fenômeno das drogas, como um problema tanto social quanto de saúde ${ }^{8}$.

A implicação dos processos de globalização e de desenvolvimento pode ser vista sob diferentes ângulos. 0 processo de globalização oferece uma interconexão entre a "vida individual" e o "futuro global" como um efeito direto ou indireto das mudanças transnacionais ocorridas nos setores econômicos, sociais e tecnológicos que os países desenvolvidos como os em desenvolvimento estão vivenciando. Isto contribui para que os aspectos das dimensões nacionais e internacionais da saúde e do processo de desenvolvimento estejam cada vez mais interligados ${ }^{10}$.

Muitos dos problemas predominantes, atuais e futuros de saúde serão globais, ou, no mínimo, multinacional ou internacional de natureza. A globalização é uma resposta ao desafio dos políticos e dos praticantes de saúde pública para criar relações entre a globalização e a saúde, enquanto 0 fenômeno das drogas se torna mais complexo ${ }^{11-12}$. Estes resultados nos levam a inferir que ainda não atingimos patamares positivos de conhecimentos sobre o fenômeno das drogas numa dimensão mais global, apesar de ser este um dos aspectos mais importantes para compreendermos a dimensão que a questão implica em todo o mundo.

0 dados relativos às relações feitas entre o fenômeno das drogas e as questões econômicas, políticas, sociais e culturais em âmbito global mostram que predominaram 117 $(65,0 \%)$ dos estudantes que concordaram com a afirmativa, ao mesmo tempo em que $34(19,0 \%)$ acadêmicos discordaram e $30(16,0 \%)$ foram indiferentes.

Os resultados evidenciam que foram feitos nexos entre 0 fenômeno das drogas e as questões econômicas, políticas, sociais e culturais durante a graduação. Tais associações se revestem de grande importância para a formação do enfermeiro, na medida em que se sabe que hoje a droga se constitui em um dos componentes da economia mundial e como tal é determinante de fatores de ordem política, econômica, social e cultural.

Esta perspectiva reforça a necessidade de se discutir conjuntamente os conteúdos sobre as políticas internacionais, a saúde internacional, globalização, tanto nos aspectos econômicos como nos sociais e culturais, considerando que estes temas estão socialmente imbricados ${ }^{8}$.
A visão micro do fenômeno das drogas considera as dimensões das questões econômicas, políticas, sociais, religiosas e antidrogas, assim como a saúde em geral. Os dados referentes a esta visão do fenômeno das drogas encontram-se expostos na Tabela 2.

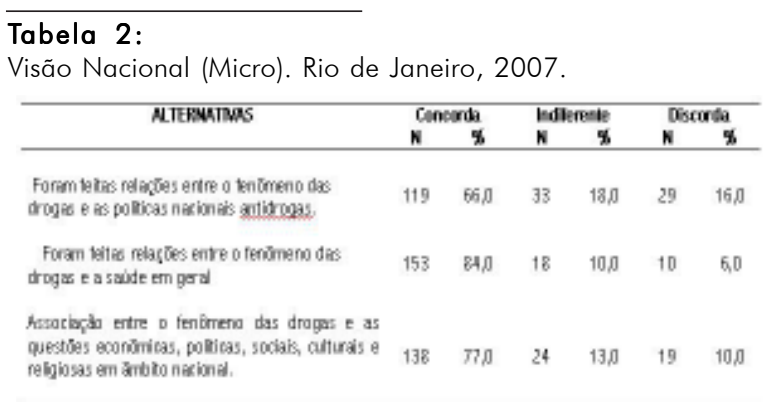

Analisando-se as relações feitas entre o fenômeno das drogas e as políticas e programas nacionais sobre drogas, $119(66,0 \%)$ acadêmicos concordam que são feitas associações entre estas variáveis no curso de graduação, enquanto 33 (18,0\%) foram indiferentes à afirmação e 29 (16,0\%) discordam.

0 Programa Nacional Antidrogas (PNAD) tem como uma de suas ações básicas priorizar a prevenção do uso indevido de drogas por ser esta a intervenção mais eficaz e de menor custo para a sociedade ${ }^{13}$.

Corroborando a afirmativa anterior, a VI Reunião Internacional do Projeto de Enfermagem da CICAD enfatizou a atuação do enfermeiro no que se refere à prevenção do uso e do abuso de drogas no contexto de família, comunidade ou ambiente de trabalho, chamando a atenção para os desafios da contemporaneidade, que demandam uma nova abordagem na formação e no treinamento daqueles que atuam nesse campo, exigindo estratégias inovadoras e modelos conceituais integrados, privilegiando o desenvolvimento de novas lideranças para trabalharem nessa área ${ }^{13}$.

Com isso, a Comissão Interamericana de Controle e Abuso de Drogas da Organização dos Estados Americanos (CICAD/OEA) vem atuando em cooperação junto a instituições governamentais e não-governamentais com o objetivo de desenvolver planos nacionais de controle de drogas e viabilizar a formação de recursos humanos para lidar com o fenômeno das drogas ${ }^{14}$.

0 Brasil apresenta uma política nacional antidrogas criada em 1998, abrangendo as áreas de redução de oferta e demanda, medidas de controle, estrutura institucional, orçamento e um sistema de avaliação. 0 conhecimento dessas esferas pelo enfermeiro é fundamental, uma vez que constitui elemento primordial no delineamento e na implementação de políticas de promoção da saúde e programas de identificação de fatores de risco do uso de drogas ${ }^{13}$.

Observou-se um percentual de $66 \%$ de estudantes (equivalente a 119) que concordam que durante a sua formação profissional houve discussão acerca das relações entre 0 fenômeno das drogas e as políticas nacionais antidrogas, o que demonstra um certo domínio por parte dos docentes das estratégias nacionais antidrogas. Para dar conta de um problema com a dimensão das drogas, é preciso compreender o fenômeno em suas diretrizes e políticas, tanto em nível internacional como em nível nacional e local. 
Os dados referentes às relações entre o fenômeno das drogas e a saúde em geral evidenciam que $153(84,0 \%)$ dos acadêmicos investigados concordam que foram feitas associações entre o fenômeno das drogas e as questões da saúde em âmbito geral, ao mesmo tempo em que 18 (10,0\%) foram indiferentes e $10(6,0 \%)$ discordaram.

Os desafios de cuidar da saúde em geral e lidar com problemas relacionados ao fenômeno das drogas, especificamente, exige parcerias e novas aproximações à educação da saúde e outros profissionais. As enfermeiras estão em uma posição única de fornecer liderança ao encontrarem desafios na saúde que afetam populações ao redor do globo, incluindo os desafios da saúde relacionados aos problemas das drogas ${ }^{14}$.

A predominância foi equivalente a $138(77,0 \%)$ estudantes entrevistados que concordaram haver associação entre o fenômeno das drogas e as políticas econômicas, sociais, culturais, e aspectos religiosos, em âmbito nacional, enquanto $19(10,0 \%)$ dos estudantes discordaram e $24(13,0 \%)$ dos acadêmicos não apresentaram uma posição diante da questão, tornando-se indiferentes.

Tais resultados demonstram que os estudantes de Enfermagem, futuros profissionais e também responsáveis pelo desenvolvimento de programas referentes às drogas, consideram-se embasados quanto às políticas (sócioeconômica-culturais) e à influência dos aspectos religiosos, condições fundamentais para implementar ações de enfermagem com os adictos. Estes resultados são discordantes de estudos feitos anteriormente sobre a formação do enfermeiro e o fenômeno das drogas ${ }^{4-5}$.

A visão pontual do fenômeno das drogas corresponde às associações entre o fenômeno das drogas e as políticas e programas nacionais/regionais sobre drogas feitas durante a graduação, às associações feitas durante as aulas entre a atuação do enfermeiro e o fenômeno das drogas, além do interesse dos estudantes concernente ao tema, evidenciado na Tabela 3.

\section{Tabela 3:}

Visão Local (Pontual). Rio de Janeiro, 2007.

\begin{tabular}{|c|c|c|c|c|c|c|}
\hline \multirow[t]{2}{*}{ MTESQTIMS } & \multicolumn{2}{|c|}{ Cencrita } & \multicolumn{2}{|c|}{ Edfeneste } & \multicolumn{2}{|c|}{ Oscturda } \\
\hline & N & 8 & k & 8 & k & $\%$ \\
\hline 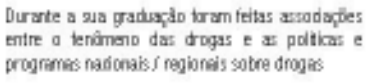 & 124 & 69,0 & 23 & 15,0 & 30 & 16,0 \\
\hline 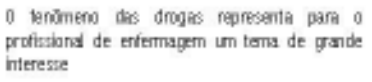 & 170 & 94,0 & 7 & 4,0 & 4 & 2,0 \\
\hline 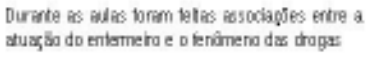 & 134 & 74,0 & 22 & $12 \pi$ & 25 & 14,0 \\
\hline
\end{tabular}

A análise da associação entre o fenômeno das drogas e as políticas e programas nacionais/regionais sobre drogas revela que a maioria dos alunos, $124(69,0 \%)$, concordou que durante a graduação foram feitas associações, enquanto $30(16,0 \%)$ discordaram e 27 (15,0\%) foram indiferentes à afirmação.

Os estudantes consideram que este conteúdo é importante na formação do enfermeiro, destacando a magnitude relacional entre as políticas e os programas regionais e nacionais. Assim entende-se que a universidade precisa incorporar o desafio de ampliar seus conteúdos sobre o fenômeno das drogas, formando profissionais capazes de estabelecer estas associações em seus campos de atuaçãa $0^{15}$.

A quase totalidade da amostra, ou seja, $170(94,0 \%)$ acadêmicos, considera o fenômeno das drogas um tema de grande interesse para o profissional de enfermagem, no entanto, 7 (4,0\%) foram indiferentes e $4(2,0 \%)$ discordaram, dado bastante relevante, visto que os enfermeiros, ao serem adequadamente capacitados para lidarem com o fenômeno das drogas, tornam-se instrumentos-chave para trabalhar a prevenção do uso de drogas e promoção da saúde ${ }^{16}$.

Em contrapartida, a falta de conhecimentos espećíicos do aluno de Enfermagem sobre o uso e abuso de drogas pode contribuir para preconceitos e estereótipos dos dependentes, os quais conseqüentemente se manifestarão nos cuidados ao paciente ${ }^{17}$.

Entretanto, $134(74,0 \%)$ estudantes concordam que durante as aulas foram feitas associações entre o fenômeno das drogas e a atuação do enfermeiro, 25 (14,0\%) discordaram da afirmação e 22 (12\%) posicionaram-se de forma indiferente.

É importante ressaltar que a atuação do enfermeiro deve se inserir num novo contexto, representado pela globalização, no qual os fatores que influenciam e condicionam a saúde não são apenas resultados de determinantes locais, mas sim de questões em escala mundial. Dessa maneira, a formação do enfermeiro deve subsidiar os conhecimentos necessários para o discernimento dos diversos fatores associados ao processo de saúde e doença ${ }^{17}$.

Entre as profissões da saúde, a Enfermagem combinou as perspectivas biofisiológica, psicossocial e populacional para fomentar liderança que será necessária nos sistemas de cuidado da saúde emergente, particularmente na luta contra o uso ilegal de drogas em todas as fases do processo. Neste sentido, enfermeiras pesquisadoras fizeram contribuiç̃̃es únicas e independentes à saúde pública ${ }^{14}$.

A Enfermagem possui uma visão ampla da saúde por enfatizar atividades relacionadas à promoção e prevenção da saúde e integração social, assim como um compromisso de cuidar e confortar. A base do conhecimento de enfermagem está fundamentada nos princípios das comunicações, educação/ saúde/paciente, formação de equipe, negociações, saúde comunitária, gerenciamento interdisciplinar e interacional. Tais enfoques no processo inter-relacional tornam as enfermeiras especialmente eficazes no apoio ao paciente ${ }^{14}$.

\section{CONCLUSÃO}

Os resultados do estudo foram claros em relação às posições assumidas pelos estudantes. Como ponto forte do estudo, destaca-se o conhecimento dos futuros enfermeiros em relação ao fenômeno das drogas como de importância fundamental para o exercício da profissão e à incorporação do cuidado às pessoas envolvidas com substâncias psicoativas como inerente ao papel do enfermeiro, acreditando no seu potencial para atuar junto a esses clientes. Este entendimento deve-se ao fato de o fenômeno das drogas ser abordado nos cursos de graduação, o que subsidiou a manifestação dos alunos sobre a temática em suas diferentes dimensões. 
Somando-se a este, encontra-se o fato de que, quanto à visão micro, os estudantes, em sua maioria, concordam com as afirmativas propostas, o que para nós sugere um domínio razoável das teorias correspondentes à relação entre o fenômeno das drogas e as políticas nacionais antidrogas, questões econômicas, políticas, sociais, culturais e religiosas em âmbito nacional, e, principalmente, a associação entre o fenômeno das drogas e a saúde em geral, demonstrando que os estudantes não vêm 0 fenômeno das drogas como um problema unicamente de saúde mental, mas como algo que envolve a totalidade do indivíduo.

A análise dos dados permite concluir que houve a incorporação de conteúdos sobre o fenômeno das drogas e os aspectos nacionais. Entretanto, em relação aos aspectos internacionais, a abordagem não se evidenciou com a mesma propriedade, o que pressupõe que os docentes ainda não conseguiram avançar no olhar e no conhecimento para a questão das drogas como um fenômeno que vai além das fronteiras do país. Neste aspecto, chama a atenção o fato de os alunos declararem não terem recebido estas informações e também não se posicionarem em relação à questão, assumindo uma posição de indiferença.

\section{Referências}

1. Luz RJP. 0 poder estratégico do conhecimento. In: El papel de las escuelas de enfermería en el área de la reducción de la demanda de drogas en América Latina. Florianópolis (SC): PEN /UFSC/; 2003.

2. Berger PL. A constituição social da realidade: tratado de sociologia do conhecimento (por) Peter L. Berger e Thomas Luckman; Tradução de Floriano de Souza Fernandes. Petrópolis(RJ): Vozes; 1976. 247p.

3. Rockeach M. Crenças, atitudes e valores. Tradução de Ângela Maria Magnan Barbosa. Revisão Técnica de Helmuth Ricardo Kruger. Rio de Janeiro(RJ): Interciência;1981.

4. Lopes, GT, Carraro TE. A formaç̧ão do enfermeiro e o fenômeno das drogas: conhecimentos, atitudes e crenças - estudo multicêntrico desenvolvido no Rio de Janeiro e região sul do Brasil. Relatório de Pesquisa. EERP/USP. Pós-Doutorado; 2004.

5. Pillon SC. 0 uso do álcool e a educação formal dos enfermeiros. [tese de doutorado]. São Paulo(SP): EPM /UNIFESP; 2003.

6. Carraro TE, Santos SMA, Fenili RM, Radunz V. 0 fenômeno das drogas e as macro-politicas: agências e tratados internacionais. Texto \& Contexto Enferm 2004. Abr/jun; 13 (2): 290-95.

7. Godue C. La salud internacional e las escuelas de Salud Publica en los Estados Unidos. In: Salud Internacional: un debate norte-sur. OPS/OMS. Washington, DC(USA); 1992.

8. Wright MGM, Spricigo JS. A saúde internacional e a problemática da droga na América Latina: uma contribuição conceitual. In: Reibnitz KS, Horr L, Souza ML, Spricigo IS, organizadores. 0 processo de cuidar, ensinar e aprender o fenômeno das drogas: políticas de saúde, educação e enfermagem: módulo 2. Florianópolis(SC): UFSC; 2003. p.43-60).

9. Frenk J, Chacón F. Bases conceptuales para la educación e investigación en salud internacional. In: Salud internacional: un debate norte-sur. OPS/OMS. Washington,DC(USA); 1992. 24p.

10. Yach D, Bettcher D. The globalization of public health, 1: threats and opportunities. Am J Public Health 1998; 88(5):735-38.
Este domínio das teorias relativas à visão nacional se reflete na visão pontual dos estudantes, que, também em sua maioria, concordaram com as afirmativas propostas, fato esse que pode implicar na formação de profissionais de enfermagem esclarecidos quanto à correspondência entre sua atuação e o fenômeno das drogas, podendo então proporcionar uma melhor qualidade no atendimento a pacientes que estejam envolvidos com drogas.

Deste modo, o espaço de produção de conhecimentos - A UNIVERSIDADE - não pode ignorar os apelos que emergem da sociedade em que está inserida. Precisa estar atenta para corresponder a estas demandas sociais, preparando os profissionais para intervir nestes cenários.

Como sugestão oriunda desta investigação, apontamos a continuidade de estudos envolvendo a formação do enfermeiro no que concerne ao fenômeno das drogas, para agregar maiores conhecimentos sobre a temática, incremento dos conteúdos relativos à contextualização internacional do fenômeno das drogas e maior sedimentação dos conhecimentos até agora adquiridos pelos estudantes.

11. Kaul I, Faust M. Global public goods and health: taking the agenda forward. World Health Organization 2001; 79(9): 869-74.

12. Woodward D, Drager N, Beaglehoke R, Lipson D. Globalization and health: a framework for analysis and action. World Health Organization 2001; 79(9): 875-81.

13. Organização dos Estados Americanos. Comission Interamericanapara para el Control de Abuso de Drogas. Relatório Hemisférico 1999/2000. Mecanismo de avaliação multilateral. Washington, DC(USA); 2000.27p.

14. Wright MG, Caufield C, Gray G, Olson J. Programas internacionais de capacitação en investigação para enfermeiros no estudo de fenômeno das drogas na América Latina: desafíos e perspectivas. Ribeirão Preto 2005; nov/dez; 13(n esp).

15. Lopes GT. A formação do enfermeiro e o fenômeno das drogas no Estado do Rio de Janeiro-Brasil: conhecimentos, atitudes e crenças. Relatório de Pesquisa apresentado ao $2^{\circ}$ Programa Regional de Formación em Investigación Aplicado al Estúdio del Fenômeno de las Drogas para Docentes de Enfermería de América Latina. Ribeirão Preto(SP): CICAD/OEA; 2004.

16. Associação Brasileira de Enfermagem-ABEn. A contribuição da profissão de enfermagem na implementação do Plano Nacional Antidrogas no Brasil: uma proposta em construção. Brasília (DF); 2002. 79p.

17. Sá MS, Moura CFS, Lopes GT. 0 conhecimento dos graduandos de enfermagem sobre o fenômeno das drogas: estudo realizado no Rio de Janeiro. Enferm Atual 2005 maio/jun; (27). 